\title{
Riemann-Hilbert problems, matrix orthogonal polynomials and discrete matrix equations with singularity confinement
}

\author{
Giovanni A. Cassatella-Contra ${ }^{\dagger}$ and Manuel Mañas ${ }^{\ddagger}$ \\ Departamento de Física Teórica II \\ (Métodos Matemáticos de la Física) \\ Universidad Complutense de Madrid \\ 28040-Madrid, Spain \\ ${ }^{\dagger}$ gaccontra@fis.ucm.es, ${ }^{\ddagger}$ manuel.manas@fis.ucm.es
}

\begin{abstract}
In this paper matrix orthogonal polynomials in the real line are described in terms of a Riemann-Hilbert problem. This approach provides an easy derivation of discrete equations for the corresponding matrix recursion coefficients. The discrete equation is explicitly derived in the matrix Freud case, associated with matrix quartic potentials. It is shown that, when the initial condition and the measure are simultaneously triangularizable, this matrix discrete equation possesses the singularity confinement property, independently if the solution under consideration is given by recursion coefficients to quartic Freud matrix orthogonal polynomials or not.
\end{abstract}

\section{Introduction}

The study of singularities of the solutions of nonlinear ordinary differential equations and, in particular, the quest of equations whose solutions are free of movable critical points, the so called Painlevé property, lead, more than 110 years ago, to the Painlevé transcendents, see [1] (and [2] for a recent account of the state of the art in this subject). The Painlevé equations are relevant in a diversity of fields, not only in Mathematics but also, for example in Theoretical Physics and in particular in 2D Quantum Gravity an Topological Field Theory, see for example [2].

A discrete version of the Painlevé property, the singularity confinement property, was introduced for the first time by Grammaticos, Ramani and Papageorgiou in 1991 [3], when they studied some discrete equations, including the dPI equation (discrete version of the first Painlevé equation), see also the contribution of these authors to [2]. For this equation they realized that if eventually a singularity could appear at some specific value of the discrete independent variable it would disappear 
after performing few steps or iterations in the equation. This property, as mentioned previously, is considered by these authors as the equivalent of the Painlevé property [1] for discrete equations. Hietarinta and Grammaticos also derived some discrete versions of the other five Painlevé equations [4, 5]. See also the interesting papers [6] and [7].

Freud orthogonal polynomials in the real line [8] are associated to the weight

$$
w_{\rho}(x)=|x|^{\rho} e^{-|x|^{m}}, \quad \rho>-1, \quad m>0 .
$$

Interestingly, for $m=2,4,6$ it has been shown [9] that from the recursion relation

$$
x p_{n}=a_{n+1} p_{n+1}(x)+b_{n} p_{n}(x)+a_{n} p_{n-1}(x),
$$

the orthogonality of the polynomials leads to a recursion relation satisfied by the recursion coefficients $a_{n}$. In particular, for $m=4$ Van Assche obtains for $a_{n}$ the discrete Painlevé I equations, and therefore its singularities are confined. For related results see also [10]. For a modern and comprehensive account of this subject see the survey [11].

In 1992 it was found [12] that the solution of a $2 \times 2$ Riemann-Hilbert problem can be expressed in terms of orthogonal polynomials in the real line and its Cauchy transforms. Later on this property has been used in the study of certain properties of asymptotic analysis of orthogonal polynomials and extended to other contexts, for example for the multiple orthogonal polynomials of mixed type [13].

Orthogonal polynomials with matrix coefficients on the real line have been considered in detail first by Krein [14, 15] in 1949, and then were studied sporadically until the last decade of the XX century. These are some papers of this subject: Berezanskii (1968) [16], Geronimo [17] (1982), and Aptekarev and Nikishin [18] (1984). In the last paper they solved the scattering problem for a kind of discrete Sturm-Liouville operators that are equivalent to the recurrence equation for scalar orthogonal polynomials. They found that polynomials that satisfy a recurrence relation of the form

$$
x P_{k}(x)=A_{k} P_{k+1}(x)+B_{k} P_{k}(x)+A_{k-1}^{*} P_{k-1}(x), \quad k=0,1, \ldots
$$

are orthogonal with respect to a positive definite measure. This is a matricial version of Favard's theorem for scalar orthogonal polynomials. Then, in the 1990's and the 2000's some authors found that matrix orthogonal polynomials (MOP) satisfy in certain cases some properties that satisfy scalar valued orthogonal polynomials; for example, Laguerre, Hermite and Jacobi polynomials, i.e., the scalar-type Rodrigues' formula [19, 20, 21] and a second order differential equation [22, 23, 24].

Later on, it has been proven [25] that operators of the form $D=\partial^{2} F_{2}(t)+\partial^{1} F_{1}(t)+\partial^{0} F_{0}$ have as eigenfunctions different infinite families of MOP's. Moreover, in [24] a new family of MOP's satisfying second order differential equations whose coefficients do not behave asymptotically as the identity matrix was found. See also [26].

The aim of this paper is to explore the singularity confinement property in the realm of matrix orthogonal polynomials. For that aim following [12] we formulate the matrix Riemann-Hilbert problem associated with the MOP's. From the Riemann-Hilbert problem it follows not only the recursion relations but also, for a type of matrix Freud weight with $m=4$, a nonlinear recursion relation (58) 
for the matrix recursion coefficients, that might be considered a matrix version -non Abelian- of the discrete Painlevé I. Finally, we prove that this matrix equation possesses the singularity confinement property, and that after a maximum of 4 steps the singularity disappears. This happens when the quartic potential $V$ and the initial recursion coefficient are simultaneously triangularizable. It is important to notice that the recursion coefficients for the matrix orthogonal Freud polynomials provide solutions to (58) and therefore the singularities are confined. A relevant fact for this solution is that the collection of all recursion coefficients is an Abelian set of matrices. However, not all solutions of (58) define a commutative set; nevertheless, the singularity confinement still holds. In this respect we must stress that our singularity confinement proof do not rely in matrix orthogonal polynomials theory but only on the analysis of the discrete equation. This special feature is not present in the scalar case previously studied elsewhere.

The layout of this paper is as follows. In section 2 the Riemann-Hilbert problem for matrix orthogonal polynomials is derived and some of its consequences studied. In $\S 3$ a discrete matrix equation, for which the recursion coefficients of the Freud MOP's are solutions, is derived and it is also proven that its singularities are confined. Therefore, it might be considered as a matrix discrete Painlevé I equation.

\section{Riemann-Hilbert problems and matrix orthogonal poly- nomials in the real line}

\subsection{Preliminaries on monic matrix orthogonal polynomials in the real line}

A family of matrix orthogonal polynomials (MOP's) in the real line [11] is associated with a matrixvalued measure $\mu$ on $\mathbb{R}$; i. e., an assignment of a positive semi-definite $N \times N$ Hermitian matrix $\mu(X)$ to every Borel set $X \subset \mathbb{R}$ which is countably additive. However, in this paper we constraint ourself to the following case: given an $N \times N$ Hermitian matrix $V(x)=\left(V_{i, j}(x)\right)$, we choose $\mathrm{d} \mu=\rho(x) \mathrm{d} x$, being $\mathrm{d} x$ the Lebesgue measure in $\mathbb{R}$, and with the weight function specified by $\rho=\exp (-V(x))$ (thus $\rho$ is a positive semi-definite Hermitian matrix). Moreover, we will consider only even functions in $x, V(x)=V(-x)$; in this situation the finiteness of the measure $\mathrm{d} \mu$ is achieved for any set of

polynomials $V_{i, j}(x)$ in $x^{2}$. Associated with this measure we have a unique family $\left\{P_{n}(x)\right\}_{n=0}^{\infty}$ of monic matrix orthogonal polynomials

$$
P_{n}(z)=\mathbb{I}_{N} z^{n}+\gamma_{n}^{(1)} z^{n-1}+\cdots+\gamma_{n}^{(n)} \in \mathbb{C}^{N \times N},
$$

such that

$$
\int_{\mathbb{R}} P_{n}(x) x^{j} \rho(x) \mathrm{d} x=0, \quad j=0, \ldots, n-1 .
$$

Here $\mathbb{I}_{N}$ denotes the identity matrix in $\mathbb{C}^{N \times N}$. 
In terms of the moments of the measure $\mathrm{d} \mu$,

$$
m_{j}:=\int_{\mathbb{R}} x^{j} \rho(x) \mathrm{d} x \in \mathbb{C}^{N \times N}, \quad j=0,1, \ldots
$$

we define the truncated moment matrix

$$
m^{(n)}:=\left(m_{i, j}\right) \in \mathbb{C}^{n N \times n N},
$$

with $m_{i, j}=m_{i+j}$ and $0 \leq i, j \leq n-1$. Invertibility of $m^{(n)}$, i.e. $\operatorname{det} m^{(n)} \neq 0$, is equivalent to the existence of a unique family of monic matrix orthogonal polynomials. In fact, we can write (1) as

$$
\left(\begin{array}{ccc}
m_{0} & \cdots & m_{n-1} \\
\vdots & & \vdots \\
m_{n-1} & \cdots & m_{2 n-2}
\end{array}\right)\left(\begin{array}{c}
\gamma_{n}^{(n)} \\
\vdots \\
\gamma_{n}^{(1)}
\end{array}\right)=\left(\begin{array}{c}
-m_{n} \\
\vdots \\
-m_{2 n-1}
\end{array}\right),
$$

and hence uniqueness is equivalent to $\operatorname{det} m^{(n)} \neq 0$. From the uniqueness and evenness we deduce that

$$
P_{n}(z)=\mathbb{I}_{N} z^{n}+\gamma_{n}^{(2)} z^{n-2}+\gamma_{n}^{(4)} z^{n-4}+\cdots+\gamma_{n}^{(n)},
$$

where $\gamma_{n}^{(n)}=0$ if $n$ is odd.

The Cauchy transform of $P_{n}(z)$ is defined by

$$
Q_{n}(z):=\frac{1}{2 \pi \mathrm{i}} \int_{\mathbb{R}} \frac{P_{n}(x)}{x-z} \rho(x) \mathrm{d} x,
$$

which is analytic for $z \in \mathbb{C} \backslash \mathbb{R}$. Recalling $\frac{1}{z-x}=\frac{1}{z} \sum_{j=0}^{n-1} \frac{x^{j}}{z^{j}}+\frac{1}{z} \frac{\left(\frac{x}{z}\right)^{n}}{1-\frac{x}{z}}$ and (1) we get

$$
Q_{n}(z)=-\frac{1}{2 \pi \mathrm{i}} \frac{1}{z^{n+1}} \int_{\mathbb{R}} \frac{P_{n}(x) x^{n}}{1-\frac{x}{z}} \rho(x) \mathrm{d} x,
$$

and consequently

$$
Q_{n}(z)=c_{n}^{-1} z^{-n-1}+O\left(z^{-n-2}\right), \quad z \rightarrow \infty,
$$

where we have introduced the coefficients

$$
c_{n}:=\left(-\frac{1}{2 \pi \mathrm{i}} \int_{\mathbb{R}} P_{n}(x) \rho(x) x^{n} \mathrm{~d} x\right)^{-1},
$$

relevant in the sequel of the paper.

Proposition 1. We have that $c_{n}$ satisfies

$$
\operatorname{det} c_{n}=-2 \pi \mathrm{i} \frac{\operatorname{det}\left(m^{(n)}\right)}{\operatorname{det}\left(m^{(n+1)}\right)} .
$$


Proof. To prove it just define $\boldsymbol{m}:=\left(m_{n}, \ldots, m_{2 n-1}\right)$, consider the identity

$$
\left(\begin{array}{cc}
m^{(n)^{-1}} & 0 \\
0 & \mathbb{I}_{N}
\end{array}\right) m^{(n+1)}=\left(\begin{array}{cc}
\mathbb{I}_{n N} & m^{(n)^{-1}} \boldsymbol{m} \\
\boldsymbol{m}^{t} & m_{2 n}
\end{array}\right)
$$

and apply the Gauss elimination method to get

$$
\frac{\operatorname{det}\left(m^{(n+1)}\right)}{\operatorname{det}\left(m^{(n)}\right)}=\operatorname{det}\left(m_{2 n}-\boldsymbol{m}^{t} m^{(n)^{-1}} \boldsymbol{m}\right) \neq 0 ;
$$

from (2) we conclude $m_{2 n}-\boldsymbol{m}^{t} m^{(n)^{-1}} \boldsymbol{m}=\int_{\mathbb{R}} P_{n}(x) x^{n} \rho(x) \mathrm{d} x$.

The evenness of $V$ leads to $Q_{n}(z)=(-1)^{n+1} Q_{n}(-z)$, so that

$$
Q_{n}(z)=c_{n}^{-1} z^{-n-1}+\sum_{j=2}^{\infty} a_{n}^{(2 j-1)} z^{-n-2 j+1}, \quad z \rightarrow \infty
$$

In particular,

$$
Q_{0}(z)=c_{0}^{-1} z^{-1}+c_{1}^{-1} z^{-3}+O\left(z^{-5}\right), \quad z \rightarrow \infty
$$

Finally, if we assume that $V_{i, j}$ are Hölder continuous we get the Plemelj formulae

$$
\left.\left(Q_{n}(z)_{+}-Q_{n}(z)_{-}\right)\right|_{x \in \mathbb{R}}=P_{n}(x) \rho(x)
$$

with $Q_{n}(x)_{+}=\left.Q_{n}(z)\right|_{z=x+i 0^{+}}$and $Q_{n}(x)_{-}=\left.Q_{n}(z)\right|_{z=x+i 0^{-}}$.

\subsection{Riemann-Hilbert problem}

Definition 1. The Riemann-Hilbert problem to consider here is the finding of a $2 N \times 2 N$ matrix function $Y_{n}(z) \in \mathbb{C}^{2 N \times 2 N}$ such that

1. $Y_{n}(z)$ is analytic in $z \in \mathbb{C} \backslash \mathbb{R}$.

2. Asymptotically behaves as

$$
Y_{n}(z)=\left(\mathbb{I}_{2 N}+O\left(z^{-1}\right)\right)\left(\begin{array}{cc}
\mathbb{I}_{N} z^{n} & 0 \\
0 & \mathbb{I}_{N} z^{-n}
\end{array}\right), \quad z \rightarrow \infty .
$$

3. On $\mathbb{R}$ we have the jump

$$
Y_{n}(x)_{+}=Y_{n}(x)_{-}\left(\begin{array}{cc}
\mathbb{I}_{N} & \rho(x) \\
0 & \mathbb{I}_{N}
\end{array}\right)
$$


An easy extension of the connection among orthogonal polynomials in the real line with a particular Riemann-Hilbert problem discovered in [12] can be proven in this matrix context.

Proposition 2. The unique solution to the Riemann-Hilbert problem specified in Definition 1 is given in terms of monic matrix orthogonal polynomials with respect to $\rho(x) \mathrm{d} x$ and its Cauchy transforms:

$$
Y_{n}(z)=\left(\begin{array}{cc}
P_{n}(z) & Q_{n}(z) \\
c_{n-1} P_{n-1}(z) & c_{n-1} Q_{n-1}(z)
\end{array}\right), \quad n \geq 1 .
$$

Proof. In the first place let us show that $\left(\begin{array}{cc}P_{n}(z) & Q_{n}(z) \\ c_{n-1} P_{n-1}(z) & c_{n-1} Q_{n-1}(z)\end{array}\right)$ does satisfy the three conditions requested by Definition 1 .

1. As the matrix orthogonal polynomials $P_{n}$ are analytic in $\mathbb{C}$ and its Cauchy transforms are analytic in $\mathbb{C} \backslash \mathbb{R}$, the proposed solution is analytic in $\mathbb{C} \backslash \mathbb{R}$.

2. Replacing the asymptotics of the matrix orthogonal polynomials and its Cauchy transforms we get $\left(\begin{array}{cc}P_{n}(z) & Q_{n}(z) \\ c_{n-1} P_{n-1}(z) & c_{n-1} Q_{n-1}(z)\end{array}\right) \rightarrow\left(\begin{array}{cc}z^{n}+O\left(z^{n-1}\right) & O\left(z^{-n-1}\right) \\ O\left(z^{n-1}\right) & z^{-n}+O\left(z^{-n-1}\right)\end{array}\right)=\left(\mathbb{I}_{2 N}+O\left(z^{-1}\right)\right)\left(\begin{array}{cc}\mathbb{I}_{N} z^{n} & 0 \\ 0 & \mathbb{I}_{N} z^{-n}\end{array}\right)$ when $z \rightarrow \infty$.

3. From 111) we get $\left(\begin{array}{cc}P_{n}(x+\mathrm{i} 0) & Q_{n}(x+\mathrm{i} 0) \\ c_{n-1} P_{n-1}(x+\mathrm{i} 0) & c_{n-1} Q_{n-1}(x+\mathrm{i} 0)\end{array}\right)-\left(\begin{array}{cc}P_{n}(x-\mathrm{i} 0) & Q_{n}(x-\mathrm{i} 0) \\ c_{n-1} P_{n-1}(x-\mathrm{i} 0) & c_{n-1} Q_{n-1}(x-\mathrm{i} 0)\end{array}\right)=\left(\begin{array}{cc}0 & P_{n}(x) \rho(x) \\ 0 & c_{n-1} P_{n-1}(x) \rho(x)\end{array}\right)$.

Then, a solution to the RH problem is $Y_{n}=\left(\begin{array}{cc}P_{n}(z) & Q_{n}(z) \\ c_{n-1} P_{n-1}(z) & c_{n-1} Q_{n-1}(z)\end{array}\right)$. But the solution is unique, as we will show now. Given any solution $Y_{n}$, its determinant $\operatorname{det} Y_{n}(z)$ is analytic in $\mathbb{C} \backslash \mathbb{R}$ and satisfies

$$
\begin{aligned}
\operatorname{det} Y_{n}(x)_{+} & =\operatorname{det}\left(Y_{n}(x)_{-}\left(\begin{array}{cc}
\mathbb{I}_{N} & \rho(x) \\
0 & \mathbb{I}_{N}
\end{array}\right)\right)=\operatorname{det} Y_{n}(x)_{-} \operatorname{det}\left(\begin{array}{cc}
\mathbb{I}_{N} & \rho(x) \\
0 & \mathbb{I}_{N}
\end{array}\right) \\
& =\operatorname{det} Y_{n}(x)_{-} .
\end{aligned}
$$

Hence, $\operatorname{det} Y_{n}(z)$ is analytic in $\mathbb{C}$. Moreover, Definition 1 implies that

$$
\operatorname{det} Y_{n}(z)=1+O\left(z^{-1}\right), \quad z \rightarrow \infty
$$

and Liouville theorem ensures that

$$
\operatorname{det} Y_{n}(z)=1, \quad \forall z \in \mathbb{C}
$$

From 15 we conclude that $Y_{n}^{-1}$ is analytic in $\mathbb{C} \backslash \mathbb{R}$. Given two solutions $Y_{n}$ and $\tilde{Y}_{n}$ of the RH problem we consider the matrix $\tilde{Y}_{n} Y_{n}^{-1}$, and observe that from property 3 of Definition 1 we have $\left(\tilde{Y}_{n} Y_{n}^{-1}\right)_{+}=\left(\tilde{Y}_{n} Y_{n}^{-1}\right)_{-}$, and consequently $\tilde{Y}_{n} Y_{n}^{-1}$ is analytic in $\mathbb{C}$. From Definition 1 we get $Y_{n} Y_{n}^{-1} \rightarrow$ $\mathbb{I}_{2 N}$ as $z \rightarrow \infty$, and Liouville theorem implies that $\tilde{Y}_{n} Y_{n}^{-1}=\mathbb{I}_{2 N}$; i.e., $\tilde{Y}_{n}=Y_{n}$ and the solution is unique. 
Definition 2. Given the matrix $Y_{n}$ we define

$$
S_{n}(z):=Y_{n}(z)\left(\begin{array}{cc}
\mathbb{I}_{N} z^{-n} & 0 \\
0 & \mathbb{I}_{N} z^{n}
\end{array}\right) .
$$

Proposition 3. 1. The matrix $S_{n}$ has unit determinant:

$$
\operatorname{det} S_{n}(z)=1 .
$$

2. It has the special form

$$
S_{n}(z)=\left(\begin{array}{cc}
A_{n}\left(z^{2}\right) & z^{-1} B_{n}\left(z^{2}\right) \\
z^{-1} C_{n}\left(z^{2}\right) & D_{n}\left(z^{2}\right)
\end{array}\right) .
$$

3. The coefficients of $S_{n}$ admit the asymptotic expansions

$$
\begin{array}{ll}
A_{n}\left(z^{2}\right)=\mathbb{I}_{N}+S_{n, 11}^{(2)} z^{-2}+O\left(z^{-4}\right), & B_{n}\left(z^{2}\right)=S_{n, 12}^{(1)}+S_{n, 12}^{(3)} z^{-2}+O\left(z^{-4}\right), \\
C_{n}\left(z^{2}\right)=S_{n, 21}^{(1)}+S_{n, 21}^{(3)} z^{-2}+O\left(z^{-4}\right), & D_{n}\left(z^{2}\right)=\mathbb{I}_{N}+S_{n, 22}^{(2)} z^{-2}+O\left(z^{-4}\right),
\end{array}
$$

for $z \rightarrow \infty$.

Proof. 1. Is a consequence of (15) and (16).

2. It follows from the parity of $P_{n}$ and $Q_{n}$.

3. 12) implies the following asymptotic behaviour

$$
S_{n}(z)=\mathbb{I}_{2 N}+S_{n}^{(1)} z^{-1}+O\left(z^{-2}\right), \quad z \rightarrow \infty,
$$

and $(18)$ gives

$$
S_{n}^{(2 i)}=\left(\begin{array}{cc}
S_{n, 11}^{(2 i)} & 0 \\
0 & S_{n, 22}^{(2 i)}
\end{array}\right), \quad \quad S_{n}^{(2 i-1)}=\left(\begin{array}{cc}
0 & S_{n, 12}^{(2 i-1)} \\
S_{n, 21}^{(2 i-1)} & 0
\end{array}\right),
$$

and the result follows.

Observe that from (18) we get

$$
S_{n}^{-1}(z)=\left(\begin{array}{cc}
\tilde{A}_{n}\left(z^{2}\right) & z^{-1} \tilde{B}_{n}\left(z^{2}\right) \\
z^{-1} \tilde{C}_{n}\left(z^{2}\right) & \tilde{D}_{n}\left(z^{2}\right)
\end{array}\right),
$$

with the asymptotic expansions for $z \rightarrow \infty$

$$
\begin{aligned}
& \tilde{A}_{n}\left(z^{2}\right)=\mathbb{I}_{N}+\left(S_{n, 12}^{(1)} S_{n, 21}^{(1)}-S_{n, 11}^{(2)}\right) z^{-2}+O\left(z^{-4}\right), \\
& \tilde{B}_{n}\left(z^{2}\right)=-S_{n, 12}^{(1)}-\left(S_{n, 12}^{(3)}-S_{n, 11}^{(2)} S_{n, 12}^{(1)}+S_{n, 12}^{(1)}\left(S_{n, 21}^{(1)} S_{n, 12}^{(1)}-S_{n, 22}^{(2)}\right)\right) z^{-2}+O\left(z^{-4}\right), \\
& \tilde{C}_{n}\left(z^{2}\right)=-S_{n, 21}^{(1)}+\left(-S_{n, 21}^{(3)}+S_{n, 21}^{(1)} S_{n, 11}^{(2)}+\left(S_{n, 22}^{(2)}-S_{n, 21}^{(1)} S_{n, 12}^{(1)}\right) S_{n, 21}^{(1)}\right) z^{-2}+O\left(z^{-4}\right), \\
& \tilde{D}_{n}\left(z^{2}\right)=\mathbb{I}_{N}+\left(S_{n, 21}^{(1)} S_{n, 12}^{(1)}-S_{n, 22}^{(2)}\right) z^{-2}+O\left(z^{-4}\right) .
\end{aligned}
$$




\subsubsection{Recursion relations}

We now introduce the necessary elements, within the Riemann-Hilbert problem approach, to derive the recursion relations and properties of the recursion coefficients in the context of matrix orthogonal polynomials.

Definition 3. We introduce the matrix

$$
Z_{n}(z):=Y_{n}(z)\left(\begin{array}{cc}
\rho(z) & 0 \\
0 & \mathbb{I}_{N}
\end{array}\right)=\left(\begin{array}{cc}
P_{n}(z) \rho(z) & Q_{n}(z) \\
c_{n-1} P_{n-1}(z) \rho(z) & c_{n-1} Q_{n-1}(z)
\end{array}\right) .
$$

Proposition 4. 1. $Z_{n}(z)$ is analytic on $\mathbb{C} \backslash \mathbb{R}$,

2. for $z \rightarrow \infty$ it holds that

$$
Z_{n}(z)=\left(\mathbb{I}_{2 N}+O\left(z^{-1}\right)\right)\left(\begin{array}{cc}
z^{n} \rho(z) & 0 \\
0 & z^{-n} \mathbb{I}_{N}
\end{array}\right)
$$

3. over $\mathbb{R}$ it is satisfied

$$
Z_{n}(x)_{+}=Z_{n}(x)_{-}\left(\begin{array}{cc}
\mathbb{I}_{N} & \mathbb{I}_{N} \\
0 & \mathbb{I}_{N}
\end{array}\right)
$$

Definition 4. We introduce

$$
\begin{aligned}
M_{n}(z) & :=\frac{\mathrm{d} Z_{n}(z)}{\mathrm{d} z} Z_{n}^{-1}(z), \\
R_{n}(z) & :=Z_{n+1}(z) Z_{n}^{-1}(z)=Y_{n+1}(z) Y_{n}^{-1}(z) .
\end{aligned}
$$

We can easily show that

Proposition 5. The matrices $M_{n}$ and $R_{n}$ satisfy

$$
M_{n+1}(z) R_{n}(z)=\frac{\mathrm{d}}{\mathrm{d} z} R_{n}(z)+R_{n}(z) M_{n}(z) .
$$

Proof. It follows from the compatibility condition

$$
T \frac{\mathrm{d} Z_{n}(z)}{\mathrm{d} z}=\frac{\mathrm{d}}{\mathrm{d} z} T Z_{n}(z)
$$

where $T F_{n}:=F_{n+1}$.

We can also show that 
Proposition 6. For the functions $R_{n}(z)$ and $M_{n}(z)$ we have the expressions

$$
\begin{aligned}
R_{n}(z) & =\left(\begin{array}{cc}
z \mathbb{I}_{N} & -S_{n, 12}^{(1)} \\
S_{n+1,21}^{(1)} & 0
\end{array}\right), \\
M_{n}(z) & \left.=\left[\begin{array}{cc}
A_{n}\left(z^{2}\right) \frac{\mathrm{d} \rho(z)}{\mathrm{d} z} \rho^{-1}(z) \tilde{A}_{n}\left(z^{2}\right) & A_{n}\left(z^{2}\right) z^{-1} \frac{\mathrm{d} \rho(z)}{\mathrm{d} z} \rho^{-1}(z) \tilde{B}_{n}\left(z^{2}\right) \\
z^{-1} C_{n}\left(z^{2}\right) \frac{\mathrm{d} \rho(z)}{\mathrm{d} z} \rho^{-1}(z) \tilde{A}_{n}\left(z^{2}\right) & z^{-2} C_{n}\left(z^{2}\right) \frac{\mathrm{d} \rho(z)}{\mathrm{d} z} \rho^{-1}(z) \tilde{B}_{n}\left(z^{2}\right)
\end{array}\right)\right]_{+},
\end{aligned}
$$

where $[\cdot]_{+}$denotes the part in positive powers of $z$.

Proof. The expression for $R_{n}$ is a consequence of the following reasoning:

1. In the first place notice that $R_{n}(z)$ is analytic for $z \in \mathbb{C} \backslash \mathbb{R}$.

2. Moreover, denoting

$$
\begin{aligned}
& R_{n+}(x):=Y_{n+1+}(x)\left(Y_{n+}(x)\right)^{-1}, \\
& R_{n-}(x):=Y_{n+1-}(x)\left(Y_{n-}(x)\right)^{-1},
\end{aligned}
$$

and substituting 13$)$ in $(30)$ we get $R_{n_{+}}(x)=R_{n_{-}}(x)$ and therefore $R_{n}(z)$ is analytic in $\mathbb{C}$.

3. Finally, if we substitute 16 in $(26)$ we deduce that

$$
\begin{aligned}
R_{n}(z) & =Y_{n+1}(z) Y_{n}^{-1}(z) \\
& =S_{n+1}(z)\left(\begin{array}{cc}
z \mathbb{I}_{N} & 0 \\
0 & z^{-1} \mathbb{I}_{N}
\end{array}\right) S_{n}^{-1}(z) \\
& =\left(\begin{array}{cc}
z \mathbb{I}_{N} & 0 \\
0 & 0
\end{array}\right)+S_{n+1}^{(1)}\left(\begin{array}{cc}
\mathbb{I}_{N} & 0 \\
0 & z^{-1} \mathbb{I}_{N}
\end{array}\right)-\left(\begin{array}{cc}
\mathbb{I}_{N} & 0 \\
0 & 0
\end{array}\right) S_{n}^{(1)}+O\left(z^{-1}\right), \quad z \rightarrow \infty,
\end{aligned}
$$

and the analyticity of $R_{n}$ in $\mathbb{C}$ leads to the desired result.

For the expression for $M_{n}$ we have the argumentation

1. $M_{n}(z)$ is analytic for $z \in \mathbb{C} \backslash \mathbb{R}$.

2. Given

$$
\begin{aligned}
& M_{n+}(x):=\frac{\mathrm{d} Z_{n+}(x)}{\mathrm{d} z}\left(Z_{n+}(x)\right)^{-1}, \\
& M_{n_{-}}(x):=\frac{\mathrm{d} Z_{n-}(x)}{\mathrm{d} z}\left(Z_{n-}(x)\right)^{-1} .
\end{aligned}
$$

Substituting (24) in 32 we get

$$
M_{n+}(x)=M_{n-}(x),
$$

and therefore $M_{n}(z)$ is analytic over $\mathbb{C}$. 
3. From 16 and 22 we see that $Z_{n}(z)$ is

$$
Z_{n}(z)=S_{n}(z)\left(\begin{array}{cc}
z^{n} \rho(z) & 0 \\
0 & z^{-n} \mathbb{I}_{N}
\end{array}\right)
$$

so that

$$
\frac{\mathrm{d} Z_{n}(z)}{\mathrm{d} z} Z_{n}^{-1}(z)=\frac{\mathrm{d} S_{n}(z)}{\mathrm{d} z} S_{n}(z)^{-1}+S_{n}(z) K_{n}(z) S_{n}^{-1}(z)
$$

where

$$
K_{n}(z):=\left(\begin{array}{cc}
n z^{-1} \mathbb{I}_{N}+\frac{\mathrm{d} \rho(z)}{\mathrm{d} z} \rho^{-1}(z) & 0 \\
0 & -n z^{-1} \mathbb{I}_{N}
\end{array}\right)
$$

Finally, as $M_{n}(z)$ is analytic over $\mathbb{C}$, (35) leads to

$$
M_{n}(z)=\frac{\mathrm{d} Z_{n}(z)}{\mathrm{d} z} Z_{n}^{-1}(z)=\left[S_{n}(z)\left(\begin{array}{cc}
\frac{\mathrm{d} \rho(z)}{\mathrm{d} z} \rho^{-1}(z) & 0 \\
0 & 0
\end{array}\right) S_{n}^{-1}(z)\right]_{+} .
$$

Observe that the diagonal terms of $M_{n}$ are odd functions of $z$ while the off diagonal are even functions of $z$. Now we give a parametrization of the first coefficients of $S$ in terms of $c_{n}$.

Proposition 7. The following formulae hold true

$$
\begin{array}{ll}
S_{n, 12}^{(1)}=c_{n}^{-1}, & S_{n, 21}^{(1)}=c_{n-1}, \\
S_{n, 11}^{(2)}=-\sum_{i=1}^{n} c_{i}^{-1} c_{i-1}+c_{n}^{-1} c_{n-1}, & S_{n, 22}^{(2)}=\sum_{i=1}^{n} c_{i-1} c_{i}^{-1}, \\
S_{n, 21}^{(3)}=-c_{n-1} \sum_{i=1}^{n-1} c_{i}^{-1} c_{i-1}+c_{n-2}, & S_{n, 12}^{(3)}=c_{n}^{-1} \sum_{i=1}^{n+1} c_{i-1} c_{i}^{-1} .
\end{array}
$$

Proof. Equating the expressions for $Y_{n}(z)$ provided by (14) and (16) we get

$$
\begin{aligned}
Y_{n}(z) & =\left(\begin{array}{cc}
P_{n}(z) & Q_{n}(z) \\
c_{n-1} P_{n-1}(z) & c_{n-1} Q_{n-1}(z)
\end{array}\right) \\
& =\left(\begin{array}{cc}
z^{n} \mathbb{I}_{N} & 0 \\
0 & z^{-n} \mathbb{I}_{N}
\end{array}\right)\left(\mathbb{I}_{2 N}+S_{n}^{(1)} z^{-1}+S_{n}^{(2)} z^{-2}+S_{n}^{(3)} z^{-3}+O\left(z^{-4}\right)\right), \quad z \rightarrow \infty .
\end{aligned}
$$


Expanding the r.h.s. we get

$$
\begin{aligned}
& S_{n, 21}^{(1)}=c_{n-1}, \quad S_{n, 12}^{(1)}=c_{n}^{-1}, \\
& S_{1,11}^{(2)}=0, \\
& S_{1,21}^{(3)}=S_{2,21}^{(3)}=0, \quad S_{n, 21}^{(3)}=c_{n-1} S_{n-1,11}^{(2)}, \quad n \geq 2, \\
& S_{n, 12}^{(3)}=c_{n}^{-1} S_{n+1,22}^{(2)},
\end{aligned}
$$

where we have used that

$$
S_{1,22}^{(2)}=c_{0} c_{1}^{-1},
$$

which can be proved from (10). Introducing (37) into 28) we get

$$
R_{n}(z)=\left(\begin{array}{cc}
z \mathbb{I}_{N} & -c_{n}^{-1} \\
c_{n} & 0
\end{array}\right),
$$

and (16) and (39) lead to

$$
S_{n+1}(z)=\left(\begin{array}{cc}
z \mathbb{I}_{N} & -c_{n}^{-1} \\
c_{n} & 0
\end{array}\right) S_{n}(z)\left(\begin{array}{cc}
z^{-1} \mathbb{I}_{N} & 0 \\
0 & z \mathbb{I}_{N}
\end{array}\right),
$$

so that

$$
\begin{aligned}
S_{n+1,11}^{(2)}-S_{n, 11}^{(2)} & =-c_{n}^{-1} c_{n-1}, \\
S_{n, 12}^{(3)}-c_{n}^{-1} S_{n, 22}^{(2)} & =c_{n+1}^{-1},
\end{aligned}
$$

where we have used (37). From (37) and (41) we get

$$
S_{n+1,22}^{(2)}-S_{n, 22}^{(2)}=c_{n} c_{n+1}^{-1} .
$$

Summing up in $n$ in (40) and (42) we deduce

$$
\begin{aligned}
& \sum_{i=1}^{n-1}\left(S_{i+1,11}^{(2)}-S_{i, 11}^{(2)}\right)=-\sum_{i=1}^{n-1} c_{i}^{-1} c_{i-1}, \\
& \sum_{i=1}^{n-1}\left(S_{i+1,22}^{(2)}-S_{i, 22}^{(2)}\right)=\sum_{i=1}^{n-1} c_{i} c_{i+1}^{-1},
\end{aligned}
$$

which leads to

$$
\begin{aligned}
& S_{n, 11}^{(2)}=-\sum_{i=1}^{n} c_{i}^{-1} c_{i-1}+c_{n}^{-1} c_{n-1}, \\
& S_{n, 22}^{(2)}=\sum_{i=1}^{n} c_{i-1} c_{i}^{-1},
\end{aligned}
$$


where we have used (37) and (38). Finally (37), 43) and 44) give

$$
S_{n, 21}^{(3)}=-c_{n-1} \sum_{i=1}^{n-1} c_{i}^{-1} c_{i-1}+c_{n-2},
$$

valid for $n \geq 2$, and

$$
S_{n, 12}^{(3)}=c_{n}^{-1} \sum_{i=1}^{n+1} c_{i-1} c_{i}^{-1} .
$$

Notice that 37 gives

$$
S_{1,21}^{(3)}=0 \text {. }
$$

Proposition 8. Matrix orthogonal polynomials $P_{n}$ (and its Cauchy transforms $Q_{n}$ ) are subject to the following recursion relations

$$
P_{n+1}(z)=z P_{n}(z)-\frac{1}{2} \beta_{n} P_{n-1}(z)
$$

with the recursion coefficients $\beta_{n}$ given by

$$
\beta_{n}:=2 c_{n}^{-1} c_{n-1}, \quad n \geq 1, \quad \beta_{0}:=0 .
$$

Proof. Observe that 26 can be written as

$$
Y_{n+1}(z)=R_{n}(z) Y_{n}(z)
$$

Then, if we replace (14) and (39) into (50) we get the result.

We now show some commutative properties of the polynomials and the recursion coefficients.

Proposition 9. Let $f(z): \mathbb{C} \rightarrow \mathbb{C}^{N \times N}$ such that $[V(x), f(z)]=0 \forall(x, z) \in \mathbb{R} \times \mathbb{C}$, then

$$
\begin{aligned}
& {\left[c_{n}, f(z)\right]=\left[\beta_{n}, f(z)\right]=0,} \\
& n \geq 0 \text {, } \\
& \forall z \in \mathbb{C}, \\
& {\left[P_{n}\left(z^{\prime}\right), f(z)\right]=0,} \\
& n \geq 0 \text {, } \\
& \forall z, z^{\prime} \in \mathbb{C} \text {. }
\end{aligned}
$$

Proof. Let us suppose that for a given $m \geq 0$ we have

$$
\left[P_{m}(x), f(z)\right]=\left[P_{m-1}(x), f(z)\right]=0 .
$$

Then, recalling (7) these expressions give

$$
\left[c_{m}, f(z)\right]=\left[c_{m-1}, f(z)\right]=0,
$$


respectively. Therefore, using the recursion relations 48) and 49) we obtain

$$
\left[P_{m+1}(x), f(z)\right]=x\left[P_{m}(x), f(z)\right]-\left[c_{m}^{-1} c_{m-1} P_{m-1}(x), f(z)\right]=0
$$

This means that

$$
\left[c_{m+1}, f(z)\right]=0
$$

Hypothesis (51) holds for $m=1$, consequently $\left[c_{n}, f(z)\right]=0$ for $n=0,1, \ldots$ and (49) implies $\left[\beta_{n}, f(z)\right]=0$. Finally, as the coefficients of the matrix orthogonal $P_{n}(z)$ are polynomials in the $\beta$ 's we conclude that $\left[P_{n}\left(z^{\prime}\right), f(z)\right]=0$ for all $z, z^{\prime} \in \mathbb{C}$.

Corollary 1. Suppose that $[V(x), V(z)]=0$ for all $x \in \mathbb{R}$ and $z \in \mathbb{C}$, then

$$
\begin{aligned}
{\left[P_{n}(z), P_{m}\left(z^{\prime}\right)\right] } & =0, \quad \forall n, m \geq 0, \quad \quad z, z^{\prime} \in \mathbb{C}, \\
{\left[c_{n}, c_{m}\right] } & =0, \\
{\left[\beta_{n}, \beta_{m}\right] } & =0 .
\end{aligned}
$$

Proof. Applying Proposition 9 to $f=V$ we deduce that $\left[P_{n}\left(z^{\prime}\right), V(z)\right]=0$, so that it allows to use again Proposition 9 but now with $f=P_{n}$ and get the stated result. From (7) and (54) we deduce (55) and using (49) we get (56).

\section{A discrete matrix equation, related to Freud matrix or- thogonal polynomials, with singularity confinement}

We will consider the particular case when

$$
V(z)=\alpha z^{2}+\mathbb{I}_{N} z^{4}, \quad \alpha=\alpha^{\dagger} .
$$

Observe that $\left[V(z), V\left(z^{\prime}\right)\right]=0$ for any pair of complex numbers $z, z^{\prime}$. Hence, in this case the corresponding set of matrix orthogonal polynomials $\left\{P_{n}\right\}_{n=0}^{\infty}$, that we refer as matrix Freud polynomials, is an Abelian set. Moreover, we have

$$
\left[c_{n}, c_{m}\right]=\left[\beta_{n}, \beta_{m}\right]=\left[c_{n}, \alpha\right]=\left[\beta_{n}, \alpha\right]=0, \quad \forall n, m=0,1, \ldots
$$

In this situation we have

Theorem 1. The recursion coefficients $\beta_{n}$ (49) for the Freud matrix orthogonal polynomials determined by (57) satisfy

$$
\beta_{n+1}=n \beta_{n}^{-1}-\beta_{n-1}-\beta_{n}-\alpha, \quad n=1,2, \ldots
$$

with $\beta_{0}:=0$. 
Proof. We compute now the matrix $M_{n}$, for which we have

$$
M_{n}(z)=\left[\left(\begin{array}{cc}
-A_{n}\left(z^{2}\right)\left(2 \alpha z+4 z^{3} \mathbb{I}_{N}\right) \tilde{A}_{n}\left(z^{2}\right) & -A_{n}\left(z^{2}\right)\left(2 \alpha+4 z^{2} \mathbb{I}_{N}\right) \tilde{B}_{n}\left(z^{2}\right) \\
-C_{n}\left(z^{2}\right)\left(2 \alpha+4 z^{2} \mathbb{I}_{N}\right) \tilde{A}_{n}\left(z^{2}\right) & -C_{n}\left(z^{2}\right)\left(2 \alpha z^{-1}+4 z \mathbb{I}_{N}\right) \tilde{B}_{n}\left(z^{2}\right)
\end{array}\right)\right]_{+},
$$

and is clear that

$$
M_{n}(z)=M_{n}^{(3)} z^{3}+M_{n}^{(2)} z^{2}+M_{n}^{(1)} z+M_{n}^{(0)}
$$

with

$$
\begin{aligned}
& M_{n}^{(3)}=\left(\begin{array}{cc}
-4 \mathbb{I}_{N} & 0 \\
0 & 0
\end{array}\right), M_{n}^{(2)}=\left(\begin{array}{cc}
0 & 4 S_{n, 12}^{(1)} \\
-4 S_{n, 21}^{(1)} & 0
\end{array}\right), M_{n}^{(1)}=\left(\begin{array}{cc}
-2 \alpha-4 S_{n, 12}^{(1)} S_{n, 21}^{(1)} & 0 \\
0 & 4 S_{n, 21}^{(1)} S_{n, 12}^{(1)}
\end{array}\right), \\
& 0 \\
& M_{n}^{(0)}=\left(\begin{array}{cc}
0 & \alpha S_{n, 12}^{(1)}+4 S_{n, 12}^{(3)}+4 S_{n, 12}^{(1)}\left(S_{n, 21}^{(1)} S_{n, 12}^{(1)}-S_{n, 22}^{(2)}\right) \\
-2 S_{n, 21}^{(1)} \alpha-4 S_{n, 21}^{(3)}+4 S_{n, 21}^{(1)} S_{n, 11}^{(2)}-4 S_{n, 21}^{(1)} S_{n, 12}^{(1)} S_{n, 21}^{(1)} & 0
\end{array}\right) .
\end{aligned}
$$

Replacing (37)-(46) into 600 we get

$$
\begin{aligned}
& M_{n}^{(3)}=\left(\begin{array}{cc}
-4 \mathbb{I}_{N} & 0 \\
0 & 0
\end{array}\right), \quad M_{n}^{(2)}=\left(\begin{array}{cc}
0 & 4 c_{n}^{-1} \\
-4 c_{n-1} & 0
\end{array}\right), \quad M_{n}^{(1)}=\left(\begin{array}{cc}
-2 \alpha-4 c_{n}^{-1} c_{n-1} & 0 \\
0 & 4 c_{n-1} c_{n}^{-1}
\end{array}\right), \\
& M_{1}^{(0)}=\left(\begin{array}{cc}
0 & 4 c_{2}^{-1}+4 c_{1}^{-1} c_{0} c_{1}^{-1}+2 \alpha c_{1}^{-1} \\
-4 c_{0} c_{1}^{-1} c_{0}-2 c_{0} \alpha & 0
\end{array}\right), \\
& M_{n}^{(0)}=\left(\begin{array}{cc}
0 & 4 c_{n+1}^{-1}+4 c_{n}^{-1} c_{n-1} c_{n}^{-1}+2 \alpha c_{n}^{-1} \\
-4 c_{n-2}-4 c_{n-1} c_{n}^{-1} c_{n-1}-2 c_{n-1} \alpha & 0
\end{array}\right),
\end{aligned}
$$

The compatibility condition (27) together with (39), (60), (61), (62) and (63) gives

$$
4\left(c_{n+2}^{-1} c_{n}+c_{n+1}^{-1} c_{n} c_{n+1}^{-1} c_{n}-c_{n}^{-1} c_{n-1} c_{n}^{-1} c_{n-1}-c_{n}^{-1} c_{n-2}\right)+2 \alpha c_{n+1}^{-1} c_{n}-2 c_{n}^{-1} c_{n-1} \alpha=\mathbb{I}_{N},
$$

for $n \geq 2$ and

$$
4\left(c_{3}^{-1} c_{1}+c_{2}^{-1} c_{1} c_{2}^{-1} c_{1}-c_{1}^{-1} c_{0} c_{1}^{-1} c_{0}\right)+2 \alpha c_{2}^{-1} c_{1}-2 c_{1}^{-1} c_{0} \alpha=\mathbb{I}_{N},
$$

which can be written as

$$
\beta_{n+2} \beta_{n+1}+\beta_{n+1}^{2}-\beta_{n}^{2}-\beta_{n} \beta_{n-1}+\alpha \beta_{n+1}-\beta_{n} \alpha=\mathbb{I}_{N}
$$

for $n \geq 2$ and

$$
\beta_{3} \beta_{2}+\beta_{2}^{2}-\beta_{1}^{2}+\alpha \beta_{2}-\beta_{1} \alpha=\mathbb{I}_{N}
$$


respectively. Using the Abelian character of the set of $\beta$ 's we arrive to

$$
\begin{aligned}
& \beta_{n+2} \beta_{n+1}+\beta_{n+1}^{2}-\beta_{n}^{2}-\beta_{n} \beta_{n-1}+\alpha\left(\beta_{n+1}-\beta_{n}\right)=\mathbb{I}_{N}, \quad n=2,3, \ldots, \\
& \beta_{3} \beta_{2}+\beta_{2}^{2}-\beta_{1}^{2}+\alpha\left(\beta_{2}-\beta_{1}\right)=\mathbb{I}_{N} .
\end{aligned}
$$

Summing up in (66) from $i=2$ up to $i=n$ we obtain

$$
\sum_{i=2}^{n}\left[\beta_{i+2} \beta_{i+1}+\beta_{i+1}^{2}-\beta_{i}^{2}-\beta_{i} \beta_{i-1}+\alpha\left(\beta_{i+1}-\beta_{i}\right)\right]=\sum_{i=2}^{n} \mathbb{I}_{N},
$$

and consequently we conclude

$$
\beta_{n+2} \beta_{n+1}+\beta_{n+1} \beta_{n}+\beta_{n+1}^{2}+\alpha \beta_{n+1}=n \mathbb{I}_{N}+k, \quad n \geq 1,
$$

where

$$
k:=\beta_{2} \beta_{1}+\beta_{3} \beta_{2}+\beta_{2}^{2}+\alpha \beta_{2}-\mathbb{I}_{N}=\beta_{2} \beta_{1}+\beta_{1}^{2}+\beta_{1} \alpha,
$$

where we have used (67). We now proceed to show that $k=\mathbb{I}_{N}$. (25) implies, for $n=1$ and $z=0$,

$$
Z_{1}^{\prime}(0)=M_{1}^{(0)} Z_{1}(0)
$$

with $M_{1}^{(0)}$ given in (62). This leads to

$$
\left(\begin{array}{c}
P_{1}^{\prime}(0) \\
c_{0} P_{0}^{\prime}(0)
\end{array}\right)=M_{1}^{(0)}\left(\begin{array}{c}
P_{1}(0) \\
c_{0} P_{0}(0)
\end{array}\right) \text {. }
$$

Now, using (3) we deduce that

$$
\left(\begin{array}{c}
\mathbb{I}_{N} \\
0
\end{array}\right)=M_{1}^{(0)}\left(\begin{array}{c}
0 \\
c_{0}
\end{array}\right)
$$

which allows us to immediately deduce that

$$
\beta_{2} \beta_{1}+\beta_{1}^{2}+\beta_{1} \alpha=\mathbb{I}_{N}
$$

and consequently $k=\mathbb{I}_{N}$. Finally, we get

$$
\beta_{n+2} \beta_{n+1}+\beta_{n+1} \beta_{n}+\beta_{n+1}^{2}+\alpha \beta_{n+1}=n \mathbb{I}_{N}+\mathbb{I}_{N} .
$$

Finally, notice that (74) reads

$$
\beta_{2}=\beta_{1}^{-1}-\beta_{1}-\alpha
$$

This theorem ensures that $\beta_{1}$ fixes $\beta_{n}$ for all $n \geq 2$, and therefore $\beta_{n}=\beta_{n}\left(\beta_{1}, \alpha\right)$. Moreover, we will see now that the solutions $\beta_{n}$ not only commute with each other but also that they can be simultaneously conjugated to lower matrices. This result is relevant in our analysis of the confinement of singularities. 


\subsection{On singularity confinement}

The study of the singularities of the discrete matrix equations (58) reveals, as we will show, that they are confined; i.e. the singularities may appear eventually, however they disappear in few steps, no more than four. The mentioned singularities in (58) appear when for some $n$ the matrix $\beta_{n}$ is not invertible, that is $\operatorname{det} \beta_{n}=0$, and we can not continue with the sequence. However, for a better understanding of this situation in the discrete case we just request that $\operatorname{det} \beta_{n}$ is a small quantity so that $\beta_{n}^{-1}$ and $\beta_{n+1}$ exist, but they are very "large" matrices in some appropriate sense. To be more precise we will consider a small parameter $\epsilon$ and suppose that in a given step $m$ of the sequence we have

$$
\begin{aligned}
& \beta_{m-1}=O(1), \\
& \operatorname{det} \beta_{m-1}=O(1) \text {, } \\
& \beta_{m}=O(1) \text {, } \\
& \operatorname{det} \beta_{m}=O\left(\epsilon^{r}\right) \text {, }
\end{aligned}
$$

for $\epsilon \rightarrow 0$ and with $r \leq N-1$. In other words, we have the asymptotic expansions

$$
\begin{array}{rlrlrl}
\beta_{m-1} & =\beta_{m-1,0}+\beta_{m-1,1} \epsilon+O\left(\epsilon^{2}\right), & & \epsilon & \rightarrow 0, & \operatorname{det} \beta_{m-1,0} \neq 0, \\
\beta_{m}=\beta_{m, 0}+\beta_{m, 1} \epsilon+O\left(\epsilon^{2}\right), & & \epsilon \rightarrow 0, & \operatorname{dim} \operatorname{Ran} \beta_{m, 0}=N-r .
\end{array}
$$

We now proceed with some preliminar material. In particular we show that we can restrict the study to the triangular case.

Proposition 10. Let us suppose that $\beta_{1}$ and $\alpha$ are simultaneously triangularizable matrices; i.e., there exist an invertible matrix $M$ such that $\beta_{1}=M \phi_{1} M^{-1}$ and $\alpha=M \gamma M^{-1}$ with $\phi_{1}$ and $\gamma$ lower triangular matrices. Then, the solutions $\beta_{n}$ of (58) can be written as

$$
\beta_{n}=M \phi_{n} M^{-1}, \quad n \geq 0,
$$

where $\phi_{n}, n=0,1, \ldots$, are lower triangular matrices satisfying

$$
\phi_{n+1}=n \phi_{n}^{-1}-\phi_{n-1}-\phi_{n}-\gamma
$$

Moreover, let us suppose that for some integer $m$ the matrices $\beta_{m+1}, \beta_{m}$ and $\alpha$ are simultaneously triangularizable, then all the sequence $\left\{\beta_{n}\right\}_{n=0}^{\infty}$ is simultaneously triangularizable.

Proof. In the one hand, from (58) we conclude that $M^{-1} \beta_{2} M$ is lower triangular and in fact that $\left\{M^{-1} \beta_{n} M\right\}_{n \geq 0}$ is a sequence of lower triangular matrices. In the other hand, if for some integer $m$ the matrices $\beta_{m+1}, \beta_{m}$ and $\alpha$ are simultaneously triangularizable we have

$$
\begin{aligned}
\beta_{m+1} & =m \beta_{m}^{-1}-\beta_{m}-\beta_{m-1}-\alpha, \\
\beta_{m} & =(m-1) \beta_{m-1}^{-1}-\beta_{m-1}-\beta_{m-2}-\alpha,
\end{aligned}
$$

which implies that $\beta_{m-1}, \beta_{m-2}$ are triangularized by the same transformation that triangularizes $\beta_{m+1}, \beta_{m}$ and $\alpha$. 
The simultaneous triangularizability can be achieved, for example, when $\left[\beta_{1}, \alpha\right]=0$, as in this case we can always find an invertible matrix $M$ such that $\beta_{1}=M \phi_{1} M^{-1}$ and $\alpha=M \gamma M^{-1}$ where $\phi_{1}$ and $\gamma$ are lower triangular matrices, for example by finding the Jordan form of these two commuting matrices. This is precisely the situation for the solutions related with matrix orthogonal polynomials. Obviously, this is just a sufficient condition. From now on, and following Proposition 10, we will assume that the simultaneous triangularizability of $\alpha$ and $\beta_{1}$ holds and study the case in where $\alpha$ and all the $\beta$ 's are lower triangular matrices. Thus, we will use the splitting

$$
\begin{aligned}
\beta_{n} & =D_{n}+N_{n}, \\
\alpha & =\alpha_{D}+\alpha_{N},
\end{aligned}
$$

where $D_{n}=\operatorname{diag}\left(D_{n ; 1}, \ldots, D_{n ; N}\right)$ and $\alpha_{D}=\operatorname{diag}\left(\alpha_{D, 1}, \ldots, \alpha_{D, N}\right)$ are the diagonal parts of $\beta_{n}$ and $\alpha$, respectively and $N_{n}$ and $\alpha_{N}$ are the strictly lower parts of $\beta_{n}$ and $\alpha$, respectively. Then, (58) splits into

$$
\begin{aligned}
D_{n+1}+N_{n+1}= & n D_{n}^{-1}-D_{n-1}-D_{n}-\alpha_{D} \\
& +n \bar{N}_{n}-N_{n-1}-N_{n}-\alpha_{N},
\end{aligned}
$$

where $\bar{N}_{n}$ denotes the strictly lower triangular part $\beta_{n}^{-1}$; i.e.,

$$
\beta_{n}^{-1}=D_{n}^{-1}+\bar{N}_{n}
$$

Hence, (58) decouples into

$$
\begin{gathered}
D_{n+1}=n D_{n}^{-1}-D_{n-1}-D_{n}-\alpha_{D}, \\
N_{n+1}=n \bar{N}_{n}-N_{n-1}-N_{n}-\alpha_{N} .
\end{gathered}
$$

In this context it is easy to realize that there always exists a transformation leading to the situation in where

$$
\beta_{m, 0}=\left(\begin{array}{ccccccc}
0 & 0 & \cdots & 0 & 0 & \cdots & 0 \\
0 & 0 & \cdots & 0 & 0 & \cdots & 0 \\
\vdots & \vdots & & \vdots & \vdots & & \vdots \\
\beta_{m, 0 ; r+1,1} & \beta_{m, 0 ; r+1,2} & \cdots & \beta_{m, 0 ; r+1, r+1} & 0 & \cdots & 0 \\
\beta_{m, 0 ; r+2,1} & \beta_{m, 0 ; r+2,2} & \cdots & \beta_{m, 0 ; r+2, r+1} & \beta_{m, 0 ; r+2, r+2} & \cdots & 0 \\
\vdots & \vdots & & \vdots & \vdots & & \vdots \\
\beta_{m, 0 ; N, 1} & \beta_{m, 0 ; N, 2} & \cdots & \beta_{m, 0 ; N, r+1} & \beta_{m, 0 ; N, r+2} & \cdots & \beta_{m, 0 ; N, N}
\end{array}\right)
$$

Proposition 11. The singularities of the diagonal part are confined. More explicitly, if we assume 
that (79), 80 and (86) hold true at a given step $m$ then

$$
\begin{aligned}
D_{m+1 ; i} & =\frac{m}{\beta_{m, 1 ; i, i}} \epsilon^{-1}-\beta_{m-1,0 ; i, i}-\frac{\beta_{m, 2 ; i, i} m}{\beta_{m, 1 ; i, i}^{2}}-\alpha_{D, i}+O(\epsilon), \\
D_{m+2 ; i} & =-\frac{m}{\beta_{m, 1 ; i, i}} \epsilon^{-1}+\beta_{m-1,0 ; i, i}+\frac{\beta_{m, 2 ; i, i} m}{\beta_{m, 1 ; i, i}^{2}}+O(\epsilon), \\
D_{m+3 ; i} & =-\beta_{m, 1 ; i, i} \frac{m+3}{m} \epsilon+O\left(\epsilon^{2}\right), \\
D_{m+4 ; i} & =\frac{m \beta_{m-1,0 ; i, i}-2 \alpha_{D, i}}{m+3}+O(\epsilon),
\end{aligned}
$$

when $\epsilon \rightarrow 0$.

Proof. From (79), 80 and (86) we deduce

$$
\begin{aligned}
D_{m-1, i} & =\beta_{m-1,0 ; i, i}+\beta_{m-1,1 ; i, i} \epsilon+O\left(\epsilon^{2}\right), \\
D_{m, i} & =\beta_{m, 1 ; i, i} \epsilon+O\left(\epsilon^{2}\right),
\end{aligned}
$$

for $\epsilon \rightarrow 0$, with $i \geq r+1$. Substitution of these expressions in (84) leads to the stated formulae. For $i \leq r$ the coefficients $D_{m-1 ; i}$ and $D_{m ; i}$ are $O(1)$ as $\epsilon \rightarrow 0$, thus they do not vanish, and consequently there is confinement of singularities for the diagonal part $D_{n}$.

In what follows we will consider asymptotic expansions taking values in the set of lower triangular matrices

$$
\mathbb{T}:=\left\{T_{0}+T_{1} \epsilon+O\left(\epsilon^{2}\right), \epsilon \rightarrow 0, \quad T_{i} \in \mathfrak{t}_{N}\right\}, \quad \mathfrak{t}_{N}:=\left\{T=\left(T_{i, j}\right) \in \mathbb{C}^{N \times N}, \quad X_{i, j}=0 \text { when } i>j\right\},
$$

where $\mathfrak{t}_{N}$ is the set of lower triangular $N \times N$ matrices. The reader should notice that this set $\mathbb{T}=\mathfrak{t}_{N}[[\epsilon]]$ is a subring of the ring of $\mathbb{C}^{N \times N}$-valued asymptotic expansions; in fact is a subring with identity, the matrix $\mathbb{I}_{N}$. We will use the notation

$$
T_{i}:=\left(\begin{array}{cc}
T_{i, 11} & 0 \\
T_{i, 21} & T_{i, 22}
\end{array}\right), \quad i \geq 1
$$

where $T_{i, 11} \in \mathfrak{t}_{r}, T_{i, 22} \in \mathfrak{t}_{N-r}$ and $T_{i, 21} \in \mathbb{C}^{(N-r) \times r}$. We consider two sets of matrices determined by (86), namely

$$
\begin{aligned}
\mathfrak{k} & :=\left\{K_{0}=\left(\begin{array}{cc}
0 & 0 \\
K_{0,21} & K_{0,22}
\end{array}\right), K_{0,21} \in \mathbb{C}^{(N-r) \times r}, K_{0,22} \in \mathfrak{t}_{N-r}\right\}, \\
\mathfrak{l} & :=\left\{L_{-1}=\left(\begin{array}{ll}
L_{-1,11} & 0 \\
L_{-1,21} & 0
\end{array}\right), L_{-1,11} \in \mathfrak{t}_{r}, L_{-1,21} \in \mathbb{C}^{(N-r) \times r}\right\},
\end{aligned}
$$


and the related sets

$$
\begin{aligned}
& \mathbb{K}:=\left\{K=K_{0}+K_{1} \epsilon+O\left(\epsilon^{2}\right) \in \mathbb{T}, \quad K_{0} \in \mathfrak{k}\right\}, \\
& \mathbb{L}:=\left\{L=L_{-1} \epsilon^{-1}+L_{0}+L_{1} \epsilon+O\left(\epsilon^{2}\right) \in \epsilon^{-1} \mathbb{T}, \quad L_{-1} \in \mathfrak{l}\right\},
\end{aligned}
$$

which fulfill the following important properties.

Proposition 12. 1. Both $\mathbb{K}$ and $\epsilon \mathbb{L}$ are subrings of the ring with identity $\mathbb{T}$, however these two subrings have no identity.

2. If an element $X \in \mathbb{K}$ is an invertible matrix, then $X^{-1} \in \mathbb{L}$, and reciprocally if $X \in \mathbb{L}$ is invertible, then $X^{-1} \in \mathbb{K}$.

3. The subrings $\epsilon \mathbb{L}$ and $\mathbb{K}$ are bilateral ideals of $\mathbb{T} ;$ i.e., $\mathbb{L} \cdot \mathbb{T} \subset \mathbb{L}, \mathbb{T} \cdot \mathbb{L} \subset \mathbb{L}, \mathbb{T} \cdot \mathbb{K} \subset \mathbb{K}$ and $\mathbb{K} \cdot \mathbb{T} \subset \mathbb{K}$.

4. We have $\mathbb{L} \cdot \mathbb{K} \subset \mathbb{T}$.

Theorem 2. If $\beta_{1}$ and $\alpha$ are simultaneously triangularizable matrices then the singularities of (58) are confined. More explicitly, if for a given step $m$ the conditions (79), (80) and (86) are satisfied then

$$
\beta_{m+1}, \beta_{m+2} \in \mathbb{L}, \quad \beta_{m+3} \in \mathbb{K}, \quad \beta_{m+4} \in \mathbb{T}, \quad \operatorname{det} \beta_{m+4}=O(1), \quad \epsilon \rightarrow 0 .
$$

Proof. From (80) and (86) we conclude that $\beta_{m} \in \mathbb{K}$ and consequently $\beta_{m}^{-1} \in \mathbb{L}$. Taking into account this fact, (58) implies that $\beta_{m+1} \in \mathbb{L}$. Therefore, $\beta_{m+1}^{-1} \in \mathbb{K}$ and (58), as $\beta_{m+1} \in \mathbb{L}$, give $\beta_{m+2} \in \mathbb{L}$ and consequently $\beta_{m+2}^{-1} \in \mathbb{K}$. Iterating (58) we get

$$
\beta_{m+3}=\beta_{m}-(m+1) \beta_{m+1}^{-1}+(m+2) \beta_{m+2}^{-1} .
$$

Using the just derived facts, $\beta_{m+1}^{-1}, \beta_{m+2}^{-1} \in \mathbb{K}$, and that $\beta_{m} \in \mathbb{K}$, we deduce $\beta_{m+3} \in \mathbb{K}$ which implies $\beta_{m+3}^{-1} \in \mathbb{L}$. Finally, (58) gives $\beta_{m+4}$ as

$$
\beta_{m+4}=(m+3) \beta_{m+3}^{-1}-\beta_{m+2}-\beta_{m+3}-\alpha .
$$

We conclude that there are only two possibilities:

1. $\beta_{m+4}=O(1)$ for $\epsilon \rightarrow 0$, or

2. $\beta_{m+4} \in \mathbb{L}$.

Let us consider both possibilities separately.

1. Recalling that the diagonal part has singularity confinement, see Proposition 11, in the first case we see that $\operatorname{det} \beta_{m+4}=O(1)$ when $\epsilon \rightarrow 0$, as desired. 
2. In this second case we write $\beta_{m+4}$ as

$$
\beta_{m+4}=\beta_{m+3}^{-1} A+O(1), \quad \epsilon \rightarrow 0, \quad A:=(m+3) \mathbb{I}-\beta_{m+3} \beta_{m+2} .
$$

Observe that repeated use of (58) leads to the following expressions:

$$
\begin{aligned}
A & =\mathbb{I}+\left[(m+1) \beta_{m+1}^{-1}-\beta_{m}\right] \beta_{m+2} \\
& =k+\mathbb{I}-\left[(m+1) \beta_{m+1}^{-1}-\beta_{m}\right] \beta_{m+1} \\
& =k-m \mathbb{I}+\beta_{m} \beta_{m+1} \\
& =k-\beta_{m}\left(\beta_{m}+\beta_{m-1}+\alpha\right),
\end{aligned}
$$

where

$$
k:=\left[(m+1) \beta_{m+1}^{-1}-\beta_{m}\right]\left[(m+1) \beta_{m+1}^{-1}-\beta_{m}-\alpha\right] .
$$

From these formulae, as $\beta_{m+1}^{-1}, \beta_{m} \in \mathbb{K}$ we deduce that $k \in \mathbb{K}$ and also that $\beta_{m}\left(\beta_{m}+\beta_{m-1}+\right.$ $\alpha) \in \mathbb{K}$. Hence, we conclude that $A \in \mathbb{K}$ and from $(95)$ and Proposition 12 we deduce that $\beta_{m+4}=O(1)$ when $\epsilon \rightarrow 0$. Consequently, we arrive to a contradiction, and only possibility 1) remains.

\section{Acknowledgements}

The authors thanks economical support from the Spanish Ministerio de Ciencia e Innovación, research project FIS2008-00200. GAC acknowledges the support of the grant Universidad Complutense de Madrid. Finally, MM reckons illuminating discussions with Dr. Mattia Cafasso in relation with orthogonality and singularity confinement, and both authors are grateful to Prof. Gabriel Álvarez Galindo for several discussions and for the experimental confirmation, via MATHEMATICA, of the existence of the confinement of singularities in the $2 \times 2$ case.

\section{References}

[1] P. Painlevé, Leçons sur la théorie analytique des equations différentielles (Leçons de Stockholm, delivered in 1895), Hermann, Paris (1897). Reprinted in Evres de Paul Painlevé, vol. I, Éditions du CNRS, Paris, (1973).

[2] R. Conte (Editor), The Painlevé Property, One Century Later, Springer Verlag, New York, (1999).

[3] B. Grammaticos, A. Ramani and V. Papageorgiou, Do integrable mappings have the Painlevé property?, Phys. Rev. Lett. 67, 1825-1828 (1991). 
[4] A. Ramani, B. Grammaticos, J. Hietarinta, Discrete versions of the Painlevé equations, Phys. Rev. Lett. 67, 1829-1832 (1991).

[5] A. Ramani, D. Takahashi, B. Grammaticos, Y. Ohta, The ultimate discretisation of the Painlevé equations, Physica D, 114, Issues 3-4, 185-196 (1998).

[6] J. Hietarinta and C. Viallet, Discrete Painlevé I and singularity confinement in projective space, Chaos Solitons and Fractals 11 (2000), 29-32.

[7] S. Lafortune and A. Goriely, Singularity confinement and algebraic integrability, J. Math.l Phys. 45 (2004), 1191-1208.

[8] G. Freud, On the coefficients in the recursion formulae of orthogonal polynomials, Proc. Royal Irish Acad. A76 (1976), 1-6.

[9] W. Van Assche, Discrete Painlevé equations for recurrence coefficients of orthogonal polynomials, Proceedings of the International Conference on Difference Equations, special Functions and Orthogonal Polynomials, World Scientific (2007), 687-725.

[10] A. P. Magnus, Freud's equations for orthogonal polynomials as discrete Painlevé equations, Symmetries and Integrability of Difference Equations (Canterbury, 1996), London Math. Soc. Lecture Note Ser., 255, Cambridge University Press, 1999, pp. 228-243.

[11] David Damanik, Alexander Pushnitski, and Barry Simon, The Analytic Theory of Matrix Orthogonal Polynomials, Surveys in Approximation Theory 4, 2008. pp. 1-85 and also arXiv:0711.2703.

[12] A. S. Fokas, A. R. Its and A. V. Kitaev, The isomonodromy approach to matrix models in 2D quantum gravity, Commun. Math. Phys. 147 (1992), 395-430.

[13] E. Daems and A. B. J. Kuijlaars, Multiple orthogonal polynomials of mixed type and nonintersecting Brownian motions, J. Approx. Theory 146 (2007), 91-114.

[14] M. G. Krein, Infinite J-matrices and a matrix moment problem, Dokl. Akad. Nauk. SSSR 69 (2) (1949), 125-128.

[15] M. G. Krein, Fundamental aspects of the representation theory of hermitian operators with deficiency index (m,m), AMS Translations, Series 2, vol. 97, Providence, Rhode Island, 1971, pp. 75-143.

[16] Yu. M. Berezanskii, Expansions in eigenfunctions of self-adjoint operators, Transl. Math. Monographs 17, Amer. Math. Soc., (1968).

[17] J. S. Geronimo, Scattering theory and matrix orthogonal polynomials on the real line, Circuits Systems Signal Process 1 (1982), 471-495. 
[18] A. I. Aptekarev, E. M. Nikishin,The scattering problem for a discrete Sturm-Liouville operator, Math. USSR-Sb. 49 (1984), 325-355.

[19] A. J. Durán, F. J. Grünbaum, Orthogonal matrix polynomials, scalar-type Rodrigues' formulas and Pearson equations, Journal of Approximation Theory 134 (2005), 267-280.

[20] A. J. Durán, F. J. Grünbaum, Structural formulas for orthogonal matrix polynomials satisfying second order differential equations, I, Constr. Approx. 22 (2005), 255-271.

[21] Rodica D. Constin, Matrix valued polynomials generated by the scalar-type Rodrigues' formulas, Journal of Approximation Theory 161 (2009), 693-705.

[22] A. J. Durán, Matrix inner product having a matrix symmetric second order differential operator, Rocky Mountain Journal of Mathematics, 27 (1997), 585-600.

[23] A. J. Durán, F. J. Grünbaum, Orthogonal matrix polynomials satisfying second order differential equations, Internat. Math. Res. Notices 10 (2004), 461-484.

[24] J. Borrego, M. Castro, A. J. Durán, Orthogonal matrix polynomials satisfying differential equations with recurrence coefficients having non-scalar limits, arXiv:1102.1578v1.

[25] A. J. Durán and M. D. de la Iglesia, Second order differential operators having several families of orthogonal matrix polynomials as eigenfunctions, Int. Math. Research Notices, Vol. 2008, Article ID rnn084, 24 pages.

[26] Cantero, M.J., Moral, L. and Velázquez, L., Differential properties of matrix orthogonal polynomials, J. Concrete Appl. Math. v3 i3. 313-334. 